Periodica Mathematica Hungarica Vol. 3 (1-2), (1973), pp. 115-124.

GENERALIZED RAMSEY THEORY FOR GRAPHS, I. DIAGONAL NUMBERS

\author{
by \\ V. CHVÁTAL (Stanford) and F. HARARY (Ann Arbor) \\ Dedicated to the memory of AlFRÉD RÉNYI
}

\title{
0. Approach
}

We use the notation and terminology of [12]. The ramsey number $r(m, n)$ as traditionally studied in graph theory may be defined as the minimum number $p$ such that every graph with $p$ points which does not contain the complete graph $K_{m}$ must have $n$ independent points. Alternatively, it is the smallest $p$ for which every coloring of the lines of $K_{p}$ with two colors, green and red, contains either a green $K_{m}$ or a red $K_{n}$. Thus the diagonal ramsey numbers $r(n, n)$ can be described in terms of 2-coloring the lines of $K_{p}$ and regarding $K_{n}$ as a forbidden monochromatic subgraph without regard to color.

This viewpoint suggests the more general situation in which an arbitrary graph $G$ has a $c$-coloring of its lines and the number of monochromatic accurrences of a forbidden subgraph $F$ (or of a forbidden family of graphs) is calculated. A host of problem areas within graph theory can be subsumed under such a formulation. These include the line-chromatic number, in which the 3-point path is forbidden. The arboricity of $G$ involves forbidding all cycles. The thickness of a graph forbids the Kuratowski graphs. Complete bipartite graphs can be taken for both $G$ and $F$, and so can cubes $Q_{n}$ and $Q_{m}$.

There has long been a sentiment in graph theory that there is an intimate relationship between extremal graph theory and ramsey numbers. It does not appear possible to derive either TuRán's Theorem or Ravsey's Theorem from the other. However, extremal bipartite graph theory does in fact imply the bipartite form of RAMSEY's Theorem. The mystery behind these implications is revealed by Theorem 1 .

A combinatorial technique used by Eroős to find a lower bound for diagonal ramsey numbers $r(n, n)$ is extended to generalized ramsey numbers for arbitrary graphs and forbidden subgraphs.

\section{Introduction}

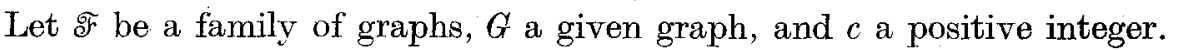
We denote by $R(G, \mathscr{F}, c)$ the greatest integer $n$ with the property that, in every coloration of the lines of $G$ with $c$ colors, there are at least $n$ monochromatic 
occurences of a member of $\mathscr{F}$. Without any loss of generality, we can assume that every forbidden subgraph $F \in \mathscr{F}$ has no isolated points. Among the typical families of of forbidden subgraphs, we mention the family $\mathfrak{C}$ of all cycles, the family $\mathcal{C}_{0}$ of odd cycles, and the family $\mathscr{T}$ of Kuratowski graphs, namely those homeomorphic to $K_{5}$ or $K_{3,3}$. If $\mathscr{F}$ contains just one forbidden subgraph $F$ then we write simply $R(G, F, c)$ instead of $R(G,\{F\}, c)$.

The numbers $R(G, \mathscr{\Im}, c)$ are useful in formulating various graph theoretical problems and results. For instance, the four color conjecture states that

$$
R(G, \mathscr{K}, 1)=0 \text { implies } R\left(G, \mathfrak{C}_{0}, 2\right)=0,
$$

i.e., that every planar graph is the line-disjoint union of two bigraphs. An equivalent formulation of the four color conjecture is:

For every bridgeless cubic planar graph $G, R\left(G, P_{3}, 3\right)=0$, in other words, every bridgeless cubic planar graph is 1 -factorable. Vizing [18] proved that

$$
R\left(G, P_{3}, \Delta+1\right)=0
$$

when he showed that the line-chromatic number of every $G$ is either $\Delta$ or $\Delta+1$. Obviously, the thickness of a graph $G$ is the minimum $n$ such that

$$
R(G, \mathfrak{d}, n)=0 .
$$

Similarly, the arboricity of $G$ is the minimum $n$ such that

$$
R(G, \mathcal{C}, n)=0 \text {. }
$$

NASH-WILliams [14] proved that the arboricity of a graph $G$ is equal to

$$
\max _{n \leqq p}\left\{\frac{q_{n}}{n-1}\right\}
$$

where $q_{n}$ is the maximum number of lines spanned by $n$ points. When Ramsey's theorem [15] is specialized to graphs, it asserts that given any positive integers $m$ and $c$ there is always an integer $n=n(m, c)$ such that

$$
R\left(K_{n}, K_{m}, c\right)>0 \text {. }
$$

Similarly, ERDős and RADO [6] proved for complete bigraphs that given any positive integers $m$ and $c$ there is always an integer $n=n(m, c)$ such that

$$
R\left(K_{n, n}, K_{m, m}, c\right)>0
$$

this is sometimes called the theorem on polarized partition relations.

We now present a brief summary of results involving $R$-numbers. ERDős and SzeKeRes [8] proved that

$$
n \geq\left(\begin{array}{r}
2 m-2 \\
m-1
\end{array}\right) \text { implies } \quad R\left(K_{n}, K_{m}, 2\right)>0,
$$


while ERDős [3] proved that

$$
m \geqq 3, \quad n \leqq 2^{m / 2} \quad \text { implies } \quad R\left(K_{n}, K_{m}, 2\right)=0,
$$

which was generalized in CHVátaL [2] to

$$
n \leqq c^{m / 2}\left(\frac{m !}{c^{1+m / 2}}\right)^{1 / m} \text { implies } R\left(K_{n}, K_{m}, c\right)=0 .
$$

GreenwoOD and Greason [10] showed that $r(4,4)=18$ by establishing that

$$
R\left(K_{17}, K_{4}, 2\right)=0 \text { and } R\left(K_{18}, K_{4}, 2\right)>0 \text {. }
$$

For a summary of other results on ramsey numbers, see [12], p. 17. Some exact results for $R$-numbers were found by Goodmax [9], who showed that

$$
R\left(K_{n}, K_{3}, 2\right)= \begin{cases}\frac{1}{3} u(u-1)(u-2) & \text { for } n=2 u \\ \frac{2}{3} u(u-1)(4 u+1) & \text { for } n=4 u+1 \\ \frac{2}{3} u(u+1)(4 u-1) & \text { for } n=4 u+3\end{cases}
$$

ERDős [4] also obtained the inequality

$$
R\left(K_{n}, K_{m}, 2\right)>\frac{2^{\left(\begin{array}{c}
n \\
m
\end{array}\right)}}{2^{\left(\begin{array}{c}
m \\
2
\end{array}\right)}}
$$

In ChVátal [1], two inequalities for bigraphs were proved:

$$
\begin{gathered}
n \geqq m c^{m} \text { implies } R\left(K_{n, n}, K_{m, m}, c\right)>0, \\
n \leqq c^{m / 2}\left(\frac{m !}{\sqrt{c}}\right)^{1 / m} \text { implies } \quad R\left(K_{n, n}, K_{m, m}, c\right)=0 .
\end{gathered}
$$

Niven, see [11], completely characterized those $a, b$ for which

$$
R\left(K_{2, m}, K_{a, b}, 2\right)=0 \text { for } m=2,3,4 .
$$

EnDős and Moon [5] derived the following limit:

$$
\lim _{m, n \rightarrow \infty} \frac{R\left(K_{m, n}, K_{a, b}, 2\right)}{\left(\begin{array}{l}
m \\
a
\end{array}\right)\left(\begin{array}{l}
n \\
b
\end{array}\right)}=\frac{2}{2^{a b}}
$$

Finally, Moon and Moser [13] obtained the inequalities

$$
R\left(K_{n, n}, K_{2,2}, 2\right) \geqq \begin{cases}2 u^{2}(u-1)(4 u-3) & \text { if } n=4 u \\ 2 u^{3}(4 u-3) & \text { if } n=4 u+1 \\ u(2 u+1)\left(4 u^{2}-u-1\right) & \text { if } n=4 u+2 \\ u^{2}(2 u+1)(4 u+3) & \text { if } n=4 u+3\end{cases}
$$


Before proceeding, we mention some simple relations satisfied by the function $R$. If $\mathscr{F}_{1}$ and $\mathscr{F}_{2}$ are families of graphs such that given any $F_{1} \in \mathscr{F}_{\bar{I}}$ there is an $F_{2} \in \mathscr{F}_{2}$ with $F_{2} \subset F_{1}$ then we write $\mathscr{\Im}_{1}<\mathscr{F}_{2}$. In particular, $\mathscr{\Im}_{1} \subset \mathscr{\mathscr { F }}_{2}$ implies $\mathscr{F}_{1}<\mathscr{F}_{2}$, and $F_{2} \subset F_{1}$ implies $\left\{F_{1}\right\}<\left\{F_{2}\right\}$. It is not difficult to see that

$$
\begin{aligned}
& G_{1} \subset G_{2}, c_{1} \geqq c_{2} \quad \text { implies } \quad R\left(G_{1}, \mathscr{F}, c\right) \leqq R\left(G_{2}, \mathscr{F}, c_{2}\right), \\
& \mathscr{\mathscr { F }}_{1}<\mathscr{F}_{2}, R\left(G, \mathscr{\digamma}_{2}, c\right)=0 \quad \text { implies } \quad R\left(G, \mathscr{\digamma}_{1}, c\right)=0 .
\end{aligned}
$$

\section{From extremal theory to line-coloring theory}

TuRÁN [17] founded extremal graph theory when he determined the greatest $q=q(p, n)$ such that not every $(p, q)$ graph contains $K_{n}$. For any family $\mathscr{F}$ of graphs, let us define ex $(p, \mathscr{F}, c)$ as the greatest $q$ such that not every $(p, q)$ graph satisfies $R(G, \mathscr{F}, c)>0$. Then for a single forbidden graph $F$, $\operatorname{ex}(p, \mathscr{o r}, 1)$ is the same as $\operatorname{ex}(p, F)$ defined in [12], p. 18. Thus the numbers determined by TURÁn are precisely ex $\left(p, K_{n}, 1\right)$. The problem of determining an asymptotic value of $\operatorname{ex}(p, F, 1)$ for an arbitrary graph $F$ was solved by Ekidős and Simonovits [7]:

$$
\lim _{p \rightarrow \infty} \frac{\operatorname{ex}(p, F, 1)}{\left(\begin{array}{l}
p \\
2
\end{array}\right)}=1-\frac{1}{\chi(F)-1} .
$$

In these terms, the well-known maximum number of lines in an acyclic graph and in a planar graph with $p \geq 3$ are given respectively by:

$$
\begin{aligned}
& \operatorname{ex}(p, \mathcal{C}, 1)=p-1, \\
& \operatorname{ex}(p, \mathscr{K}, 1)=3 p-6 .
\end{aligned}
$$

TURÁN observed that numerically, the forbidding of triangles is tantamount to the exclusion of all odd cycles:

$$
\operatorname{ex}\left(p, \mathfrak{C}_{0}, 1\right)=\operatorname{ex}\left(p, K_{3}, 1\right)=\left[\frac{p^{2}}{4}\right] .
$$

Not surprisingly, there is a monotonic relation between the function ex and all its variables:

$$
\begin{gathered}
p_{1} \leqq p_{2}, \mathscr{F}_{2}<\mathscr{F}_{1}, c_{1} \leqq c_{2} \text { implies } \operatorname{ex}\left(p_{1}, \mathscr{F}_{1}, c_{1}\right) \leqq \operatorname{ex}\left(p_{2}, \mathscr{\mathscr { F }}_{2}, c_{2}\right) \\
\operatorname{ex}\left(p, \mathscr{F}, c_{1} c_{2}\right) \leqq c_{1} \operatorname{ex}\left(p, \mathscr{F}, c_{2}\right) .
\end{gathered}
$$

The numbers $\operatorname{ex}\left(p, K_{n}, c\right)$ were investigated by Sós [16]; she observed that

$$
\operatorname{ex}\left(p, K_{n}, c\right)=\operatorname{ex}\left(p, K_{r}, 1\right) \text {, where } r=r\left(K_{n}, c\right) .
$$


The "easy half" of (2.3) follows at once from the more general inequality

$$
\operatorname{ex}(p, F, c) \leqq \operatorname{ex}(p, G, 1)
$$

which holds for any two graphs $F$ and $G$ such that $R(G, F, c)>0$.

We now state and prove a general theorem which links extremal graph theory with line-coloring.

THEOREM 1. Let $\mathscr{F}$ be a family of graphs containing a bicolorable graph $F$ and let $G_{1}, G_{2}, \ldots$ be a sequence of graphs with $p_{n}=p\left(G_{n}\right)$ and $q_{n}=q\left(G_{n}\right)$ such thai

$$
\begin{aligned}
& \lim _{n \rightarrow \infty} p_{n}=+\infty, \\
& \liminf _{n \rightarrow \infty} \frac{q_{n}}{p_{n}^{2}}>0 .
\end{aligned}
$$

Then given any positive integer $c$ there is a subscript $n_{0}=n_{0}(c)$ such that

$$
n \geq n_{0} \quad \text { implies } R\left(G_{n}, \mathfrak{F}, c\right)>0 .
$$

Proof. Let us denote the left-hand side of (2.5) by $L$. By (2.1) for some $p_{0}$,

$$
p \geq p_{0} \quad \text { implies } \quad \frac{\operatorname{ex}(p, F, 1)}{\left(\begin{array}{l}
p \\
2
\end{array}\right)}<\frac{L}{c} .
$$

By (2.4) and (2.5), there is a value $n_{0}$ such that

$$
n \geq n_{0} \text { implies } p_{n} \geq p_{0} \text { and } q_{n} / p_{n}^{2}>L / 2 .
$$

But then we have for each $n \geqq n_{0}$

$$
\operatorname{ex}\left(p_{n}, F, c\right) \leqq c \operatorname{ex}\left(p_{n}, F, 1\right)<L\left(\begin{array}{c}
p_{n} \\
2
\end{array}\right)<q_{n}
$$

and so, by the very definition of $\operatorname{ex}(p, F, c)$, we have

$$
R\left(G_{n}, F, c\right)>0 .
$$

As the singleton $\left\{F^{\prime}\right\}<\mathscr{F}$, we have by $(1.6)$,

$$
R\left(G_{n}, \mathscr{F}, c\right)>0
$$

which is the desired result.

When in Theorem 1, we take the particular families of graphs $\mathscr{F}=\left\{K_{m, m}\right\}$ and $G_{n}=K_{n, n}$, we obtain as a corollary the above mentioned theorem of ERDŐS and RADO on polarized partition relations. However, RAMSEY's theorem cannot be obtained in this way since $\operatorname{ex}\left(p, K_{n}, 1\right)$ is too large. More generally, 
the above approach is bound to fail whenever $\mathscr{F}=\{F\}$ and $\chi(F)>2$, for then by (2.1),

$$
\lim _{p \rightarrow \infty} \frac{\operatorname{ex}(p, F, 1)}{\left(\begin{array}{l}
p \\
2
\end{array}\right)} \geq \frac{1}{2}
$$

and so the method does not work. Thus the "Turanisation" of Ramsey-type theorems is fruitful if and only if the forbidden subgraphs are bicolorable.

\section{A combinatorial method for $R$-numbers}

ERDós' proof of the lower bound (1.1) for ramsey numbers is based on a combinatorial counting argument. Applying his proof technique to a more general situation with arbitrary $F$ and $c$ colors, we obtain

TheoRem 2. $\sum_{F \in F} R(G, F, 1) c^{-q(F)+1}<1$ implies $R(G, \mathfrak{F}, c)=0$.

Proof. Let us consider the set $S$ of all the line-colorings of $G$ with $c$ colors. By a bad coloring, we mean one which gives rise to a monochromatic subgraph of $G$ isomorphic to a member of $\mathscr{F}$. Let $S_{B}$ denote the bad colorings in $S$. Clearly, we have

$$
|S|=c^{q(G)}
$$

and moreover

$$
\left|S_{B}\right| \leqq \sum_{F \in \mathscr{Q}^{5}} R(G, F, 1) c c^{q(a)-q(F)}
$$

The inequality (3.2) follows from the fact that each $f \in S_{B}$ is completely determined by (a) the choice of $F \in \mathscr{F}$, (b) the choice of a subgraph $F_{B}$ isomorphic to $F$ which may occur in $R(G, F, 1)$ ways, (c) the choice of one of the colors for the lines of $F_{B}$, and also (d) the choice of any colors for the remaining $q(G)-q(F)$ lines of $G$.

If the hypothesis of the theorem holds, then the right hand side of (3.2) is strictly smaller than the right hand side of (3.1) and so we have

$$
\left|S_{B}\right|<|S|, \text { so that } S-S_{B} \neq \emptyset \text {, }
$$

and hence $R(G, \mathscr{\mathscr { F }}, c)=0$, completing the proof.

Although $R(G, F, 1)$ is often difficult to evaluate, it can be found quite easily when $G=K_{n}$.

Without any loss of generality, we can assume that every forbidden subgraph $F \in \mathscr{F}$ has no isolated points. For convenience, let $p=p(F)$, and 
let $s=s(F)$ be the order of the automorphism group of $F$, i.e., the number of symmetries of $F$. Since $F$ has no isolated points,

$$
R\left(K_{n}, F, 1\right)=\frac{n(n-1) \ldots(n-p+1)}{s}=\left(\begin{array}{l}
n \\
p
\end{array}\right) \frac{p !}{s} .
$$

Indeed, $F$ can be imbedded within $K_{n}$ in $\left(\begin{array}{l}n \\ p\end{array}\right) p$ ! ways and these imbeddings fall into equivalence classes, each of these having exactly $s$ members.

In order to express the next three corollaries of Theorem 2 compactly, we require the following notation. Given any graph $F$ and a positive integer $c$ there is always (by Ramsey's theorem) an integer $n$ with $R\left(K_{n}, F^{\prime}, c\right)>0$. The smallest such $n$ will be denoted by $r(F, c)$.

The first corollary gives a general lower bound for this number.

Corollary 2A. $r(F, c)>\left(s c^{q-1}\right)^{1 / p}$.

Proof. If $p>n$ then obviously $R\left(K_{n}, F, c\right)=0$. On the other hand, when

then we have

$$
1<p \leqq n \leqq\left(s c^{q-1}\right)^{1 / p}
$$

$$
R(G, F, 1) c^{-q+1} \leqq\left(\begin{array}{l}
n \\
p
\end{array}\right) \frac{p !}{s} c^{-q+1}<\frac{n^{p}}{s} e^{-q+1} \leqq 1
$$

and so, by Theorem $2 ; R\left(K_{t}, F, c\right)=0$ again. Therefore

$$
R\left(K_{n}, F^{\prime}, c\right)>0 \quad \text { implies } \quad n>\left(s c^{q-1}\right)^{1 / p}
$$

which is the desired result.

Setting $F=K_{m}$ in Corollary $2 \mathrm{~A}$, we obtain (1.2) as a special case. Setting $F=K_{m, m}$ we have $p=2 m, q=m^{2}, s=2(m !)^{2}$ and so, by Corollary $2 \mathrm{~A}$, we obtain an inequality for complete bigraphs corresponding to (1.2).

COROLJARY 2B.

$$
r\left(K_{m, m}, c\right)>c^{m / 2}\left(\frac{2(m !)^{2}}{c}\right)^{1 / 2 m} c^{m / 2} .
$$

To show that this inequality is not too horrible, we remark that (1.5) implies

$$
r\left(K_{m, m}, c\right) \leqq 2 m c^{m}
$$

Finally, in the case of the cube, $F=Q_{m}$, we have $p=2^{m}, q=m 2^{m-1}$, $s=m ! 2^{m}$ and so we obtain another analogous inequality.

Corollary 2C.

$$
r\left(Q_{m}, c\right)>c^{m / 2}\left(\frac{m ! 2^{m}}{c}\right)^{1 / 2 m}
$$




\section{Cubical R-numbers}

In this section, we shall evaluate $R\left(G, F^{\prime}, c\right)$ when $F$ and $G$ are both eubes. First of all, it is not difficult to calculate that

$$
R\left(Q_{n}, Q_{m}, 1\right)=\left(\begin{array}{l}
n \\
m
\end{array}\right) 2^{n-m}
$$

This can be also verified by the recursive formula

$$
R\left(Q_{n}, Q_{m+1}, 1\right)=2 R\left(Q_{n}, Q_{m}, 1\right)+R\left(Q_{n}, Q_{m-1}, 1\right)
$$

On the other hand, we will show that

$$
R\left(Q_{n}, Q_{2}, 2\right)=0 \text { for all } n
$$

To prove this, represent the points of $Q_{n}$ by subsets of $\{1,2, \ldots, n\}$; color the line $u v$ green if $\min (|u|,|v|)$ is even, color $u v$ red if $\min (|u|,|v|)$ is odd. It is easy to verify that $Q_{n}$ colored in this way contains no monochromatic $Q_{2}$.

Obviously, (4.1) implies

$$
R\left(Q_{n}, Q_{m}, c\right)=0 \quad \text { whenever } \quad \min (m, c)>1 .
$$

The only remaining case is $m=1$, when obviously

$$
R\left(Q_{n}, Q_{1}, c\right)=q\left(Q_{n}\right)=n 2^{n-1} .
$$

Summarizing these results, we obtain

Theorem 3.

$$
R\left(Q_{n}, Q_{m}, c\right)= \begin{cases}\left(\begin{array}{l}
n \\
m
\end{array}\right) 2^{n-m} & \text { if } \min (m, c)=1 \\
0 & \text { otherwise }\end{cases}
$$

\section{R-numbers for trees}

As in all of graph theory, trees are easier to handle than arbitrary graphs. Let us consider $R\left(T_{2}, T_{1}, c\right)$ where $T_{1}$ and $T_{2}$ are trees. It is easy to see that $R\left(T, P_{4}, 2\right)=0$ for any tree $T$. Thus, we have $P_{4} \subset T_{1}, c \geq 2$ implies $R\left(T_{2}, T_{1}, c\right)=0$. If $P_{4} \leftarrow T_{1}$, then $T_{1}$ is a star. It is not difficult to evaluate $R\left(K_{1, m}, K_{1, n}, c\right)$. For this purpose, let us define $M(m, n, c)$ as the minimum of 
$\sum_{i=1}^{c}\left(\begin{array}{l}x_{i} \\ m\end{array}\right)$ over all the partitions $n=\sum_{i=1}^{c} x_{i}$ into integers $x_{i}$. Evidently, when $n=a m+b$ with $0 \leqq b<n$, we have

and

$$
M(m, n, c)=b\left(\begin{array}{c}
a+1 \\
m
\end{array}\right)+(n-b)\left(\begin{array}{l}
a \\
m
\end{array}\right)=n\left(\begin{array}{l}
a \\
m
\end{array}\right)+b\left(\begin{array}{c}
a \\
m-1
\end{array}\right)
$$

$$
R\left(K_{1, n}, K_{1, m}, c\right)=M(m, n, c) .
$$

Furthermore, the lines of any tree $T$ can be colored with $c$ colors so that at each point $u$, the number of occurrences of each color on the lines of the star are almost equal.

Theorem 4. For any two trees $T_{1}$ and $T_{2}$ such that $T_{1}$ is not a star,

$$
R\left(T_{2}, T_{1}, c\right)=0 \quad \text { whenever } \quad c \geq 2 \text {. }
$$

On the other hand, if $T_{1}=K_{1, m}$ is a star and $d_{1}, d_{2}, \ldots, d_{n}$ is the degree sequence of $T_{2}$, then

$$
R\left(T_{2}, T_{1}, c\right)=\sum_{i=1}^{p} M\left(m, d_{i}, c\right)
$$

\section{Open Problems}

1. In another paper in this series, we develop corresponding theorems and numerical results for the nondiagonal case. Here we deal with families of forbidden subgraphs $\mathscr{F}_{1}, \mathscr{F}_{2}, \ldots, \mathscr{\mathscr { F }}_{c}$ and require a line-coloring of an arbitrary graph $G$ with $c$ colors to contain no member of family $\mathscr{F}_{i}$ which is monochromatic with color $i$.

2. One paper in this series, to be written jointly with P. HeLL, studies forbidden digraphs.

3. It is now also natural to investigate generalized ramsey numbers for multigraphs.

4. Recall that $r(F, c)$ is the smallest integer $p$ such that every $c$-coloring of the lines of $K_{p}$ contains a monochromatic $F$. We determine the exact values of $r(F, 2)$ for all forbidden graphs $F$ with at most four points in our next paper. This brings us to the frontier of solved problems as the calculation of the number $r\left(K_{5}, 2\right)$ has been open for decades and is still not known. 


\section{REFERENCES}

[1] V. Chvátal, On finite polarized partition relations, Canad. Math. Bull, 12 (1969), $321-326$.

[2] V. Chvátal, Hypergraphs and Ramseyian theorems, Proc. Amer. Math. Soc. (to appear).

[3] P. Erdős, Some remarks on the theory of graphs, Bull. Amer. Math. Soc. 53 (1947), $292-294$.

[4] P. ERDős, On the number of complete subgraphs contained in certain graphs, Magyar Tud. Akad. Mat. Kutató Int. Közl. 7 (1962), 459-464.

[5] P. ERDós and J. W. Moon, On subgraphs of the complete bipartite graph, Canad. Math. Bull. 7 (1964), 35-39.

[6] P. Erdós and R. Radó, Partition calculus in set theory, Bull. Amer. Math. Soc. 62 (1956), 427-489.

[7] P. ERdós and M. Srmonovits, A limit theorem in graph theory, Studia Sci. Math. Hungar. 1 (1966), 51-57.

[8] P. Erdős and G. Szerkeres, A combinatorial problem in geometry, Compositio Math. 2 (1935), 463-470.

[9] A. W. Goodman, On sets of acquaintances and strangers at any party, Amer. Math. Monthly 66 (1959), 778-783.

[10] R. E. Greenwoon and A. M. Gleason, Combinatorial relations and chromatic graphs, Canad. J. Math. 7 (1955), 1-7.

[11] R. K. GuY, A many-facetted problem of Zarankiewicz, The Many Facets of Graph Theory, New York, 1969, 129-148.

[12] F. Harary, Graph Theory, Reading, 1969.

[13] J. W. Moon and L. Moser, On chromatic bipartite graphs, Math. Mag. 35 (1962), $225-227$.

[14] C. St. J. A. Nash-Williams, Edge-disjoint spanning trees of finite graphs, $J$. London Math. Soe. 36 (1961), 445-450.

[15] F. P. Ramsey, On a problem of formal logic, Proc. London Math. Soc. 30 (1930), $264-286$.

[16] V. T. Sós, On extremal problems in graph theory, Combinatorial Structures and Their Applications, New York, 1970, 407-410.

[17] P. Turán, Egy gráfelméleti szélsỏértékfeladatról, Mat. Fiz. Lapok 48 (1941), 436-452; see also, On the theory of graphs, Colloq. Math. 3 (1954), 19-30.

[18] V. G. Vizina, On an estimate of the chromatic class of a $p$-graph, Diskret. Analiz. 3 (1964), 25-30 (in Russian).

(Received May 14, 1971)

DEPARTMENT OF COMPUTER SCIENCE

STANFORD UNIVERSITY

GTANFORD, CALIFORNIA 94305

DEPARTMENT OF MATHEMATICS

UNIVERSITY OF MICHTGAN

ANN ARBOR, MICHIGAN 48104

U.S.A. 